\title{
Espécies de plantas visitadas por Heliactin bilophus (Aves, Trochilidae) na Chapada dos Veadeiros, durante a estação chuvosa
}

\author{
Gabriel Baruffaldi Ghiringhello ${ }^{1 *}$ \\ Dárius Pukenis Tubelis ${ }^{2}$ \\ ${ }^{1}$ Centro de Ciências Médicas e Biológicas, Fundação São Paulo, PUC-SP, Campus Sorocaba CEP 18030-095, Sorocaba - SP, Brasil \\ ${ }^{2}$ Departamento de Ecologia, Instituto de Biociências, Universidade de São Paulo \\ CEP 05508-900, São Paulo - SP, Brasil \\ dtubelis@yahoo.com \\ *Autor para correspondência \\ Rua Maria Lopes Monteiro, 112, Santa Rosália \\ CEP 18095-530, Sorocaba - SP, Brasil \\ gbghiringhello@hotmail.com
}

Submetido em 04/02/2009

Aceito para publicação em 21/08/2009

\section{Resumo}

Os hábitos alimentares do beija-flor Heliactin bilophus ainda são pouco conhecidos. Este trabalho teve como objetivo identificar as espécies de plantas mais visitadas por H. bilophus com finalidades de alimentação (consumo de néctar) durante a estação chuvosa no Parque Nacional da Chapada dos Veadeiros, em Goiás. As observações foram realizadas em duas estações chuvosas (janeiro de 2006 e fevereiro de 2008). A metodologia baseou-se em percorrer trilhas em duas áreas de campo sujo e em duas de campo rupestre. Todo registro de um indivíduo consumindo néctar de uma planta em flor foi considerado como uma visita. Foram observadas 296 visitas, compreendendo oito espécies de plantas dos estratos arbustivo e herbáceo. As espécies mais frequentemente visitadas foram Bauhinia tenella (Caesalpinoideae) e Vochysia pumila (Vochysiaceae) em campo sujo, e Lychnophora ericoides (Asteraceae), em campo rupestre. Essas três espécies foram comumente encontradas nos locais de estudo. Nas duas fisionomias, Heliactin bilophus consumiu néctar de poucas espécies de plantas. As espécies mais frequentemente visitadas apresentavam flores em abundância.

Unitermos: campo rupestre, Cerrado, dieta, ecologia alimentar, néctar

\section{Abstract}

Plant species visited by the Horned Sungem Heliactin bilophus (Aves, Trochilidae) at Chapada dos Veadeiros, during the rainy season. The feeding habits of the Horned Sungem remain little known. This study aimed to identify the plant species most often visited by $H$. bilophus with feeding purposes (consumption of nectar) during the rainy season at Chapada dos Veadeiros National Park, Goiás. Observations were made during two rainy seasons (January 2006 and February 2008). The methodology consisted of walking through trails within two areas of campo sujo and two areas of campo rupestre. The record of an individual consuming the nectar of a flowering plant was considered to constitute a visit. A total of 296 visits were observed, comprising eight species of shrubs and herbs. The most often visited plant species were Bauhinia tenella (Caesalpinoideae) 
and Vochysia pumila (Vochysiaceae) in campo sujo, and Lychnophora ericoides (Asteraceae) in campo rupestre. These three species were commonly found at the study sites. In both physiognomies, Heliactin bilophus consumed the nectar of few plant species. The most frequently visited species bore high numbers of flowers.

Key words: Cerrado, diet, feeding ecology, nectar, rocky grassland

\section{Introdução}

Estudos sobre a alimentação de aves consumidoras de néctar no Cerrado podem ser divididos em dois grupos principais. Um deles engloba estudos envolvendo observações sobre espécies de aves forrageando em exemplares de uma ou poucas espécies de plantas selecionadas (e.g., Rojas e Ribon, 1997; Melo, 2001). O outro grupo inclui estudos que se baseiam na escolha de uma ou poucas espécies de aves, que têm suas atividades de forrageamento acompanhadas, levando à identificação de seus itens alimentares (e.g., Vasconcelos e Lombardi, 2001; Tubelis, 2004).

A alta riqueza de espécies de aves encontrada no Cerrado (Silva, 1995) contribui para que os hábitos alimentares de muitas espécies ainda permaneçam não examinados em detalhe. O beija-flor Heliactin bilophus (Temminck 1820) é um exemplo dessa situação.

Além de ser encontrado em áreas restritas da Bolívia e Suriname, esta espécie tem ampla distribuição no Brasil, ocorrendo por quase toda a extensão do Cerrado (Meyer de Schauensee, 1970; Ruschi, 1986; Grantsau, 1988; Stotz et al., 1996; Sick, 1997; Del Hoyo et al., 1999; Sigrist, 2006). Heliactin bilophus é uma das menores espécies de beija-flores encontradas no Brasil (Sick, 1997), habitando fisionomias abertas de regiões savânicas, sendo mais comum em campos sujos e campos limpos do que em outras fisionomias (Ruschi, 1967; Negret, 1988; Stotz et al., 1996; Tubelis e Cavalcanti, 2001; Sigrist, 2006). Suas populações podem ser residentes ou migratórias (Negret e Negret, 1981; Del Hoyo et al., 1999). No Distrito Federal, reproduz-se entre junho e outubro (Negret, 1988).

Quanto à alimentação de $H$. bilophus, existem apenas registros isolados de consumo de néctar de poucas espécies vegetais, e de insetos (e.g., Ruschi, 1959; Negret, 1988; Del Hoyo et al., 1999). Assim, não existem estudos sistemáticos sobre seus hábitos alimentares.
Este estudo teve como objetivo identificar as espécies de plantas mais visitadas por H. bilophus com finalidades de alimentação, em trechos de campo sujo e campo rupestre no Parque Nacional da Chapada dos Veadeiros, durante a estação chuvosa.

\section{Material e Métodos}

\section{Área de estudo}

Este estudo foi realizado no Parque Nacional Chapada dos Veadeiros, município de Alto Paraíso de Goiás, estado de Goiás. O parque apresenta paisagens características do Cerrado do Brasil central (Eiten, 1972; Oliveira e Marquis, 2002). Entre as fisionomias vegetais presentes neste parque e em seu entorno encontramse: campo limpo, campo sujo, campo cerrado, cerrado sensu stricto, campo rupestre, matas de galeria e veredas (Silva et al., 2001). O clima na região é marcado por dois períodos bem definidos - estação seca e outra chuvosa (Assad, 1994). A precipitação anual varia entre 1200 e $2000 \mathrm{~mm}$ e concentra-se entre outubro e março. A estação seca geralmente ocorre entre maio e setembro. Temperaturas podem atingir $0^{\circ} \mathrm{C}$ em julho e $40^{\circ} \mathrm{C}$ em janeiro e fevereiro (Assad, 1994).

\section{Locais de estudo}

Foram selecionados quatro locais de estudo em uma região do parque inacessível a turistas. Os locais de estudo se situam em uma área de $8 \mathrm{~km} 2\left(14^{\circ} 04^{\prime} \mathrm{S}\right.$ e $47^{\circ} 40^{\prime} \mathrm{W}$ ) dominada por vegetação nativa pouco alterada. Os locais de estudo estavam a mais de $300 \mathrm{~m}$ de construções (alojamentos do parque) e compreendem duas fisionomias comuns na reserva: campo rupestre $(\mathrm{n}=$ 2) e campo sujo $(n=2)$. Pequenas manchas de gramíneas exóticas, como o capim gordura Melinis spp. (Poaceae), ocorrem nos locais estudados principalmente ao longo de estradas de terra. 
Os campos rupestres apresentam numerosos afloramentos rochosos cobertos por abundantes liquens; o estrato herbáceo é razoavelmente desenvolvido. Arbustos são abundantes, enquanto árvores ocorrem em baixa densidade. Entre as espécies arbustivas mais numerosas, destacam-se a arnica-brasileira (Lychnophora ericoides Less, Asteraceae) e canelasde-ema (Vellozia spp, Velloziaceae).

Os campos sujos amostrados apresentavam denso estrato herbáceo, numerosos arbustos e árvores esparsas. A bate-caixa (Palicourea rígida Kunth, Rubiaceae) e as canelas-de-ema (Vellozia spp., Velloziaceae) são exemplos de espécies arbustivas mais comumente encontradas. Nesta fisionomia, o relevo é menos acidentado do que aquele dos campos rupestres. Informações adicionais sobre a vegetação de campos nativos deste parque podem ser encontradas em Silva et al. (2001).

\section{Observação das aves}

O trabalho de campo foi realizado por um observador (GBG) em duas estações chuvosas. Foram feitos 15 dias de trabalho de campo em janeiro de 2006 e 15 em fevereiro de 2008. As observações foram feitas no período da manhã (07:00 - 10:00h) e no período da tarde (17:00 - 19:00h). Um local de estudo foi visitado por dia. Campos sujos e rupestres foram amostrados em dias alternados, de modo a garantir que os quatro locais de estudo fossem visitados em um número similar de manhãs e tardes.

A amostragem consistiu em percorrer trechos de $400 \mathrm{~m}$ de trilhas pré-existentes nos locais de estudo. Ao serem encontrados, indivíduos de $H$. bilophus tiveram seus movimentos e comportamentos acompanhados. Todo registro de um indivíduo explorando néctar de uma planta com flores foi considerado como uma visita. A obtenção consecutiva de néctar em flores de uma mesma planta foi desconsiderada. A exploração de plantas próximas foi considerada como nova visita desde que as plantas estivessem a mais de $5 \mathrm{~m}$ uma da outra. Quando isso ocorreu, o observador deslocou-se após a obtenção de néctar em flores de cinco diferentes plantas, a fim de aumentar a independência espacial das amostras.

\section{Estimativas de abundância de plantas}

Durante a campanha de janeiro de 2006, as três espécies vegetais mais frequentemente visitadas tiveram suas abundâncias estimadas nas duas fisionomias. Para tal, seis transectos de $100 \mathrm{~m}$ de comprimento foram estabelecidos aleatoriamente em cada fisionomia (três por local de estudo). Plantas em floração situadas numa faixa de $4 \mathrm{~m}$ de largura ao longo do transecto $(2 \mathrm{~m}$ de cada lado) foram contadas. Plantas situadas a mais de $1 \mathrm{~m}$ de outros exemplares da mesma espécie foram consideradas como indivíduos diferentes.

\section{Resultados}

\section{Espécies de plantas utilizadas}

Foram registradas 296 visitas feitas por Heliactin bilophus, sendo 154 delas em janeiro de 2006 e 142 em fevereiro de 2008. Este troquilídeo explorou flores de oito espécies vegetais: Palicourea rigida Kunth (Rubiaceae), Bauhinia tenella Benth (Caesalpinoideae), Cuphea linarioides Cham. \& Schlecht. (Lythraceae), Lychnophora ericoides Less (Asteraceae), Vochysia pumila Pohl (Vochysiaceae), Psittacanthus robustus Mart (Loranthaceae), Vernonia monocephala Gardner (Asteraceae) e Dyckia sp. (Bromeliaceae). A observação de visitas às duas últimas espécies ocorreu somente em fevereiro de 2008. A curva de riqueza acumulada de espécies visitadas por H. bilophus estabilizou após a observação de cerca de 200 visitas, em fase inicial de observações no segundo ano do estudo (Figura 1). Assim, o esforço amostral foi adequado para se identificar as espécies vegetais exploradas pela espécie nos locais de estudo, no período estudado. 


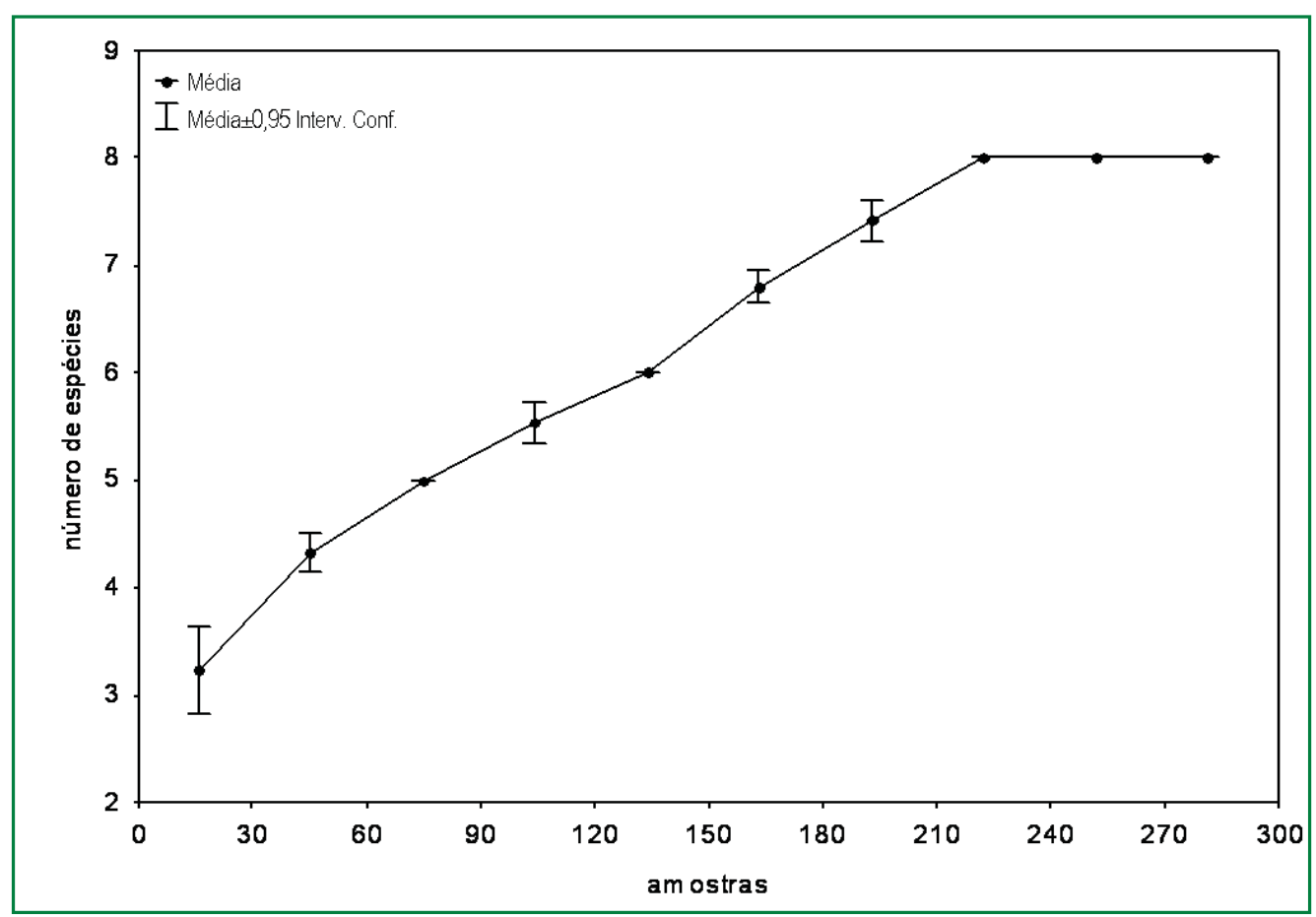

FIGURA 1: Curva acumulativa de espécies de plantas floridas visitadas por Heliactin bilophus em campos sujos e campos rupestres no Parque Nacional da Chapada dos Veadeiros, em janeiro de 2006 e fevereiro de 2008.

\section{Campos sujos}

Um total de 135 visitas foi observado em campos sujos, envolvendo quatro espécies de plantas: $B$. tenella, $V$. pumila, $P$. rigida e $V$. monocephala. Em janeiro de 2006, quando 80 visitas foram detectadas, três espécies floridas foram exploradas (Figura 2). Aquelas com maior número de visitas foram as ervas $B$. tenella $\mathrm{e} V$. pumila, representando $72,5 \%$ e $25,0 \%$ das visitas. A espécie arbustiva $P$. rigida representou os $2,5 \%$ restantes das visitas ocorridas a plantas em floração em 2006. Em fevereiro de 2008, quando 55 visitas foram observadas, novamente três espécies de plantas foram exploradas (Figura 2). A erva $V$. pumila compreendeu $89,1 \%$ das visitas. As outras duas espécies exploradas (as ervas $B$. tenella e $V$. monocephala) representaram apenas $7,3 \% \mathrm{e}$
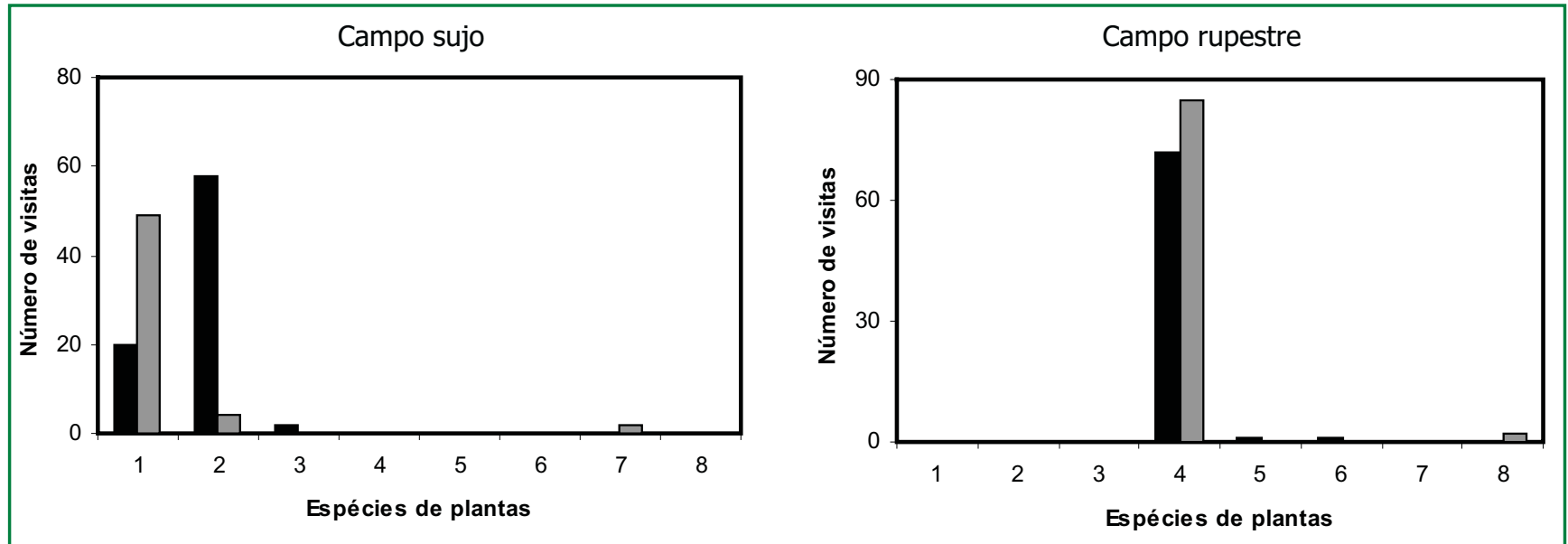

FIGURA 2: Número de visitas do beija-flor Heliactin bilophus a plantas floridas em campos sujos e em campos rupestres no Parque Nacional da Chapada dos Veadeiros em janeiro de 2006 (barras negras) e fevereiro de 2008 (barras cinzas). Espécies de plantas - 1: Vochysia pumila; 2: Bauhinia tenella; 3: Palicourea rigida; 4: Lychnophora ericoides; 5: Psittacanthus robustus; 6: Cuphea linarioides; 7 : Vernonia monocephala; 8: Dyckia sp. 
3,6\% das visitas, respectivamente (Figura 2). Em 2006, B. tenella teve mais visitas do que $V$. pumila, ao passo que esta situação se inverteu em 2008 (Figura 2).

\section{Campos rupestres}

Um total de 161 visitas às flores de quatro espécies (L. ericoides, C. linarioides, P. robustus e Dyckia sp.) foi detectado em campos rupestres. Em 2006, quando 74 visitas foram registradas, três espécies foram exploradas. A maioria (97,0\%) das visitas ocorreu em exemplares da espécie arbustiva L. ericoides (Figura 2). As outras duas espécies foram $C$. linarioides e $P$. robustus, cada uma representando apenas 1,5\% das visitas. Em 2008, quando 87 visitas foram detectadas, houve predomínio de uso de L. ericoides, compreendendo $97,7 \%$ das visitas. A outra espécie explorada neste período foi Dyckia sp., com apenas duas visitas (2,3\%). Em campos rupestres, não foram observadas diferenças marcantes na utilização de espécies vegetais por $H$. bilophus entre os dois períodos estudados.

\section{Abundância de espécies vegetais visi- tadas}

Bauhinia tenella e $V$. pumila foram comuns nos campos sujos, sendo ausentes nos campos rupestres. Por outro lado, L. ericoides foi numerosa em campos rupestres, não tendo ocorrido nos campos sujos (Tabela 1). Assim, as três espécies vegetais mais frequentemente visitadas por $H$. bilophus foram comumente encontradas, mas em apenas uma das fisionomias estudadas.

TABELA 1: Média (com desvio padrão) do número de plantas com flores, em áreas de 400 $\mathrm{m}^{2}$, das três espécies mais visitadas por Heliactin bilophus (Aves, Trochilidae) em campos sujos e campos rupestres no Parque Nacional Chapada dos Veadeiros, em janeiro de 2006.

\begin{tabular}{lcc}
\hline \multicolumn{1}{c}{ Espécie } & \multicolumn{2}{c}{ Fisionomia } \\
& campo sujo & campo rupestre \\
\hline Bauhinia tenella & $6,5(5,6)$ & 0 \\
Vochysia pumila & $3,0(2,5)$ & 0 \\
Lychnophora ericoides & 0 & $10,7(3,7)$ \\
\hline
\end{tabular}

\section{Discussão}

As oito espécies de plantas registradas neste estudo sendo exploradas por H. bilophus não haviam sido documentadas como itens alimentares desta espécie. Pesquisas anteriores realizadas em outras regiões do Cerrado mencionaram o consumo de recursos florais de arbustos e árvores dos gêneros Anacardium, Stachytarpheta, Lantana, Citrus, Hyptis, Croton, Malvaviscus e de espécies das famílias Malvaceae e Urticaceae (Ruschi, 1959; 1967; 1986; Negret e Negret, 1981; Negret, 1988; Del Hoyo et al., 1999). Assim, o presente estudo aumentou consideravelmente a lista de espécies de plantas reconhecidas como parte da dieta de H. bilophus, apesar da amostragem ter envolvido somente 30 dias de estudo, na estação chuvosa, em quatro locais de estudo.

Também, este estudo foi o primeiro a quantificar a frequência de visitação de $H$. bilophus a diferentes espécies de plantas. Nas duas fisionomias estudadas, esta espécie visitou poucas espécies vegetais e concentrou suas visitas em uma ou duas delas. Assim, as espécies frequentemente visitadas por H. bilophus (L. ericoides, $B$. tenella e $V$. pumila) deveriam ser incluídas entre as plantas consideradas como importantes para sua sobrevivência na Chapada dos Veadeiros.

Tanto em campos sujos como rupestres, indivíduos de $H$. bilophus visitaram plantas que ocorriam em alta densidade e que apresentavam numerosas flores. Assim, suas atividades alimentares aparentemente se concentram em áreas com alta disponibilidade de flores. Em várias ocasiões, indivíduos encontrados em áreas com alta densidade de L. ericoide, B. tenella ou V. pumila visitaram numerosas $(>10)$ plantas, permanecendo ali por alguns minutos. Isso foi observado ao permanecer em algumas dessas áreas, mesmo após a observação de cinco visitas, até a ave ser perdida de vista. Provavelmente, indivíduos devem considerar como prioritárias para alimentação manchas das matrizes de campos sujos e campos rupestres contendo altas concentrações dessas plantas.

Contudo, consideramos que não somente a alta concentração de flores numa dada área, mas aspectos como a disponibilidade de néctar e a morfologia floral podem influenciar a visitação a determinadas espécies 
vegetais. Isto porque, durante o período amostral, não foram registrados indivíduos de H. bilophus visitando certas espécies em floração e que eram bastante comuns nos campos estudados, como Diplusodon oblongus Pohl (Lythraceae) e Eriosema glabrum Mart (Papilionoideae). Estudos detalhados sobre aspectos (morfologia e recursos florais) das espécies vegetais são necessários para verificar a influência desses fatores sobre a exploração de diferentes espécies.

A frequência de visitação de $H$. bilophus a diferentes espécies vegetais também pode ser influenciada por interações com outras espécies de aves. Por exemplo, em duas ocasiões, indivíduos solitários de H. bilophus foram atacados por um indivíduo de Eupetomena macroura Gmelin 1788, que defendeu áreas de campo sujo com altas concentrações de B. tenella. Defesas territoriais similares por parte de E. macroura contra outras espécies de beija-flores já haviam sido registradas em outras regiões (Sick, 1997; Del Hoyo et al., 1999; Sigrist, 2006).

Em várias ocasiões, indivíduos de $H$. bilophus foram observados deixando a mancha de vegetação na qual consumiram néctar. Isto envolveu movimentos entre áreas de campos sujos e de campos rupestres adjacentes, assim como a partida de indivíduos dessas fisionomias para manchas de campo cerrado ou cerrado sensu stricto. Tais deslocamentos sugerem que áreas ocupadas por indivíduos de $H$. bilophus podem incluir várias fisionomias, mesmo num período de poucas horas ou poucos dias. Estudos sobre suas áreas de vida podem esclarecer melhor os fatores envolvidos em sua estratégia de forrageio e em sua ocorrência sazonal em paisagens do Cerrado.

Como a amostragem deste estudo foi realizada somente em janeiro e fevereiro (estação chuvosa), é essencial que sejam feitas observações em outros períodos, a fim de se compreender melhor a exploração de plantas por $H$. bilophus. Também, a condução de observações em outros horários do dia também poderia trazer novas informações.

O registro do uso de oito espécies de plantas em apenas dois meses do ano nos quatro locais de estudo sugere que a riqueza de plantas utilizadas durante o ano por $H$. bilophus pode envolver dezenas de espécies no Cerrado. A alta riqueza de espécies vegetais encontrada em campos sujos, campos cerrados, cerrados sensu stricto e campos rupestres (Mendonça et al., 1998; Silva et al., 2001) deve contribuir para tal fato. Diferenças marcantes entre as espécies mais visitadas em campos sujos e campos rupestres sugerem que estudos visando o conhecimento sobre os itens alimentares de H. bilophus devem considerar a gama de fisionomias usadas por esta espécie em paisagens do Cerrado. Seria importante identificar as espécies mais frequentemente visitadas ao longo do ciclo anual em várias partes do Cerrado.

\section{Agradecimentos}

Agradecemos ao IBAMA e a José F. S. Rebello, Daniel Borges e Simone Fonseca pela permissão de pesquisa e apoio logístico no Parque Nacional da Chapada dos Veadeiros. Margarete Naomi Sato gentilmente identificou as espécies vegetais. Teresa Cristina Anacleto, Luís Fábio Silveira e dois revisores anônimos forneceram numerosos comentários construtivos a este manuscrito. FAPESP (proc. 05/00773-3), CNPq (proc. 471360/2006-6), Idea Wild e Birders' Exchange financiaram esta pesquisa.

\section{Referências}

Assad, E. D. 1994. Chuva nos cerrados. Análise e espacialização. Embrapa/SPI, Brasília, Brasil, 423pp.

Del Hoyo, J.; Elliott, A.; Sargatal, J. 1999. Handbook of the birds of the world. v. 5. Barn-owls to Hummingbirds. Lynx Editions, Barcelona, España, 759pp.

Eiten, G. 1972. The cerrado vegetation of Brazil. Botanical Review, 38: 205-341.

Grantsau, R. 1988. Os beija-flores do Brasil. Expressão e Cultura, Rio de Janeiro, Brasil, 233pp.

Melo, C. 2001. Diurnal bird visiting of Caryocar brasiliense Camb. in central Brazil. Revista Brasileira de Biologia, 61: 311-316.

Mendonça, R. C.; Felfili, J. M.; Walter, B. M. T.; Silva Junior, M. C.; Rezende, A. V.; Filgueiras, T. S.; Nogueira, P. E. 1998. Flora vascular do Cerrado. In: Sano, S. M. \& Almeida, S. P. (Eds). Cerrado - Ambiente e flora. Embrapa, Planaltina, Brasil, p.289556.

Meyer de Schauensee, R. 1970. A guide to the birds of South America. Livingston Publ. Co., Wynnewood, USA, 577pp.

Negret, A. J. 1988. Zur Biologie der Sonnenstrahlelfe Heliactin cornuta in Zentralbrasilien. Trochilus, 9: 61-64. 
Negret, A. J.; Negret, R. A. 1981. As aves migratórias do Distrito Federal, Brasília. Boletim Técnico IBDF, 6: 1-64.

Oliveira, P. S.; Marquis, R. J. 2002. The Cerrados of Brazil. Ecology and natural history of a neotropical savanna. Columbia University Press, Nova York, USA, 398pp.

Rojas, R.; Ribon, R. 1997. Guilda de aves em Bowdichia virgilioides (Fabaceae: Faboidae) em área de cerrado de Furnas, Minas Gerais. Ararajuba, 5: 189-194.

Ruschi, A. 1959. A Trochilifauna de Brasília, com a descrição de um novo representante de Amazilia (Aves) e o primeiro povoamento com essas aves ai realizado. Boletim do Museu de Biologia Professor Mello-Leitão, Série Biológica, 22: 1-16.

Ruschi, A. 1967. Beija-flores das matas, dos scrubs, das savanas, dos campos e grasslands do Brasil, e a sua zoogeografia (Trochilidae - Aves). Boletim do Museu de Biologia Professor Mello-Leitão, Série Biológica, 51: 1-23.

Ruschi, A. 1986. Aves do Brasil. Expressão e Cultura, Rio de Janeiro, Brasil, 288pp.

Sick, H. 1997. Ornitologia brasileira. Editora Nova Fronteira, Rio de Janeiro, Brasil, 862pp.
Sigrist, T. 2006. Aves do Brasil. Uma visão artística. Birds of Brazil. An artistic view. Fosfertil, São Paulo, Brasil, 672pp.

Silva, J. M. C. 1995. Birds of the Cerrado region - South America. Steenstrupia, 21: 69-92.

Silva, S. R.; Silva, A. P.; Munhoz, C. B.; Silva Jr, M. C.; de Mederios, M. B. 2001. Guia de plantas do Cerrado utilizadas na Chapada dos Veadeiros. WWF, Brasília, Brasil, 132pp.

Stotz, D. F.; Fitzpatrick, J. W.; Parker III, T. A.; Moskovits, D. K. 1996. Neotropical birds. Ecology and conservation. Chicago University Press, Chicago, USA, 478pp.

Tubelis, D. P. 2004. Species composition and seasonal occurrence of mixed-species flocks of forest birds in savannas in central Cerrado, Brazil. Ararajuba, 12: 105-111.

Tubelis, D. P.; Cavalcanti, R. B. 2001. Community similarity and abundance of bird species in open habitats of a central Brazilian Cerrado. Ornitologia Neotropical, 12: 57-73.

Vasconcelos, M. F.; Lombardi, J. A. 2001. Hummingbirds and their flowers in the campos rupestres of southern Espinhaço Range, Brazil. Melopsittacus, 4: 3-30. 